\title{
Research in Prehospital and Disaster Health and Medicine: The Introduction Section of a Study Manuscript
}

\author{
Samuel J. Stratton, MD, MPH
}

This Editorial is a continuation of the comments presented in the last issue of Prehospital and Disaster Medicine. In that issue, the importance of developing and stating a study objective was explored. This editorial explores the importance of the Introduction Section of a scientific manuscript. The Introduction Section is usually glossed over when one reviews the instructions for authors section of a medical journal. But, a poorly written Introduction Section can distract readers and editors from taking a scientific work seriously. More important is that a careful read of an author's introduction to their work can reveal researcher bias in conduct of their study.

The International Committee of Medical Journal Editors gives the following manuscript preparation instructions for the Introduction Section of a health or medical paper:

\section{Introduction \\ Provide a context or background for the study (that is, the nature of the problem and its significance). State the specific purpose or research objective of, or hypothesis tested by, the study or observation. Cite only directly pertinent references, and do not include data of conclusions from the work being reported. ${ }^{1}$}

The Introduction Section is an essential element of a manuscript. But, both experienced and novice researchers usually find that writing a coherent and effective Introduction Section is challenging.

A well-written Introduction Section develops interest in reading and evaluating the results of the research that is presented in a manuscript. An Introduction Section should "hook" readers and other researchers into having interest in the study being presented. Additionally, the introduction provides a baseline for what is discussed in a manuscript and describes the gap(s) in knowledge that are addressed by the research presented. An important element of the Introduction Section is the statement of the study objective or hypothesis which is usually presented in the final sentence or paragraph.

An Introduction Section best begins with background information regarding the topic of the research. Often researchers will provide epidemiologic data for the disease or condition that is being studied, or will describe an event or geographic area that is the focus of the presented study. Early in an Introduction Section, definitions of major terms or words that are used in a manuscript should be provided. Starting an Introduction Section by giving principal background information helps focus both the author and reader to the primary topic for the research being presented.

In addition to background information, the basic pathophysiology or health challenge addressed by the research is appropriate to describe. Describing the pathophysiology or health challenge helps develop the statement of the study objective and to identify the outcome that is the focus for the research. For example, if research is done to study foot wounds that occur from broken glass and sharp debris following a tsunami, it helps to briefly describe the health risks of walking in debris generated from a tsunami event.

Following a presentation of background information for the research topic and discussion of the pathophysiology or health risks, a review of the work of others who have explored the same area of research is appropriate. This section of the Introduction Section should not be an exhaustive literature review. While it is essential that a researcher be familiar with all published literature regarding the topic of their research, it is not necessary to provide all literature published on the topic in the Introduction Section of a manuscript. Rather, only the most important and authoritative literature is expected and preferred. A common error in writing Introduction Sections is an attempt to provide a complete literature review. This error causes an Introduction Section to be too lengthy and detailed, often resulting in confusion about the research being presented in the manuscript. The review of previous work in the research field should be concise and present only the most important papers for the topic.

Following a concise presentation of the established literature for the research topic, it should be stated why the research being presented is important. Research may be important for different reasons, including filling gaps in knowledge for the field of interest, confirming previous research, or showing lack of effectiveness of an intervention. A major reason for an Introduction Section is to establish a justification for the research that is being described. To do this, an author must precisely present the gap in knowledge or other reason that supports the research as being an essential addition to the scientific knowledge base.

A final part of the Introduction Section is a statement of the study objective or hypothesis. This statement is customarily presented in the final paragraph of the Introduction Section and most often has best impact if presented as the last sentence of the final paragraph. More information on constructing a study objective statement is available in the previous issue of Prehospital and Disaster Medicine. ${ }^{2}$

There is no standard template for writing an Introduction Section. But, Table 1 provides a suggested structure for the Introduction Section. An Introduction Section should be concise and generally five or fewer paragraphs in length. The Introduction Section should build an argument for conducting the research being presented and state the objective for the research. Lengthy Introduction Section discussions are distracting and usually add little to the presentation of one's research. 


\section{Elements of an Introduction Section}

1. Background information, including any important definitions

2. Describe basic pathophysiology or health issue that research addresses

3. Review the work of others, providing sentinel studies in the area of research

4. State why the research is important; what knowledge gaps will the research address

5. State the study objective or hypothesis (research question to be answered)

Stratton $\odot 2014$ Prehospital and Disaster Medicine

Table 1. The Suggested Elements of a Manuscript Introduction Section

It is also important to avoid words or terms that indicate bias or opinion in an Introduction Section as well as in the entire manuscript. Table 2 lists words that are commonly associated with researcher bias. When encountering these or similar words that indicate bias, it is likely the research is flawed by researcher bias and results or findings are not valid. True scientific study is done objectively by a researcher who is seeking an answer to a research question and not trying to prove an already assumed outcome or opinion.

In summary, the Introduction Section of a scientific manuscript serves several purposes. The Introduction Section
Words that Imply Researcher Bias

Obviously
Generally
Extreme
Horrible
Tragic
Expensive
Sadly
Terrible
Lessons Learned

Stratton ๑ 2014 Prehospital and Disaster Medicine

Table 2. Avoid Use of These or Similar Words That May Indicate Researcher Bias

introduces the research topic and any key definitions. Reviews of previous work done in the specific research area are presented. Important elements of the Introduction Section are identification of why the research presented is important and a clear statement of the study objective or hypothesis. A proper Introduction Section concisely provides a background of the research area being written about, identifies the knowledge gaps that the research is designed to fill, and states the study question. In this way, the Introduction Section leads a reader to the next section of the manuscript, the Methods Section.

\section{References}

1. International Committee of Medical Journal Editors. Preparing for submission c. introduction. http://www.icmje.org/recommendations/browse/manuscript-preparation/. Accessed September 9, 2014.

2. Stratton SJ. Research in prehospital and disaster health and medicine: developing a research objective statement. Prehosp Disaster Med. 2014;29(4):341-343. 